\title{
INFLUENCIA DE LA CONCIENCIA AMBIENTAL EN LA ECOEFICIENCIA DE LAS FAMILIAS DEL DISTRITO CIUDAD NUEVA, 2019
}

\author{
INFLUENCE OF ENVIRONMENTAL AWARENESS ON THE ECOEFFICIENCY OF \\ FAMILIES IN NEW CITY DISTRICT, 2019
}

\author{
María del Pilar Torres Zapata ${ }^{1}$ \\ Karina Yanina Acosta Caipa ${ }^{2}$ \\ Richard Sabino Lazo Ramos ${ }^{3}$
}

Aceptado: $17 / 12 / 2019$

Publicado online:10/07/2020

\begin{abstract}
RESUMEN
El objetivo de la investigación fue analizar la influencia de la conciencia ambiental en la ecoeficiencia de las familias del distrito Ciudad Nueva. El horizonte temporal de la investigación correspondió al primer semestre del año 2019. Se recolecto información de 366 familias del distrito de Ciudad Nueva. La metodología utilizada fue el enfoque cuantitativo básico de alcance correlacional, siendo su diseño no experimental, retrospectivo transversal. Se encontró con una seguridad estadística del $99 \%$, que es débil y positiva $\left(r=+0,253^{* *}\right)$ la influencia de la conciencia ambiental en el uso eficiente del agua en las familias del distrito de Ciudad Nueva. Así mismo, se encontró con una seguridad estadística del $99 \%$, que es moderada y positiva $\left(r=+0,418^{* *}\right)$ la influencia de la conciencia ambiental en el uso eficiente de energía en las familias del distrito de Ciudad Nueva. Igualmente, se encontró con una seguridad estadística del $99 \%$, que es débil y positiva $\left(r=+0,355^{* *}\right)$ la influencia de la conciencia ambiental en la gestión eficiente de residuos en las familias del distrito de Ciudad Nueva. Finalmente, se encontró una correlación débil, positiva y altamente significativa entre las variables conciencia ambiental y ecoeficiencia $\left(r=+0,386^{* *}\right)$. Estos resultados permiten confirmar con una seguridad estadística del $99 \%$, que la conciencia ambiental influye positivamente en la ecoeficiencia de las familias del distrito de Ciudad Nueva.
\end{abstract}

Palabras clave: Conciencia ambiental, ecoeficiencia.

\footnotetext{
${ }_{1}^{1}$ Magister en Gestión ambiental y desarrollo sostenible, arquitecto, Gerente General de K\&M Consultores Contratistas asociados. SRL Proyectista de la Unidad formuladora del Gobierno Regional de Tacna. Perú. Arq.mptorres@gmail.com. ORCID: 0000-0002-4441-4147 2 Magister en Gestión ambiental y desarrollo sostenible, arquitecto, Gerente Comercial de K\&M Consultores Contratistas asociados. SRL. Docente de la Escuela Profesional de Arquitectura de la Universidad Jorge Basadre Grohmann de Tacna. Perú. kacostac@unjbg.edu.pe, karyacostac@gmail.com. ORCID: 0000-0003-1872-9062.

${ }^{3}$ Biólogo, Doctor en Ciencias Ambientales. Director de la Escuela Profesional de ingeniería Ambiental. Director del IDEA-UPT y del IERAUPT.acreditacion.epiam@gmail.com, ozalsomar@gmail.com ORCID: 0000-0002-7878-7486
} 


\begin{abstract}
The objective of the research was Analyze the influence of environmental awareness in the families of the Ciudad Nueva district. The time horizon of the investigation corresponds to the first semester of the year 2019. Information was gathered from 366 families from the district of Ciudad Nueva. The methodology focused on the basic quantitative approach of correlational scope, being its non-experimental, transversal retrospective design. It was found with a statistical security of $99 \%$, which is weak and positive $\left(r=+0.253^{* *}\right)$ the influence of environmental awareness on the efficient use of water in the families of the Ciudad Nueva district. Likewise, it was found with a statistical security of $99 \%$, which is moderate and positive $\left(r=+0.418^{* *}\right)$ the influence of environmental awareness on the efficient use of energy in the families of the district of Ciudad Nueva. Likewise, it was found with a statistical security of $99 \%$, which is weak and positive $\left(r=+0.355^{* *}\right)$ the influence of environmental awareness in the efficient management of waste in the families of the Ciudad Nueva district. Finally, a weak, positive and highly significant correlation was found between the variables environmental awareness and eco-efficiency ( $r=+$ $\left.0.386^{* *}\right)$. These results allow us to confirm with a statistical security of $99 \%$, that environmental awareness positively influences the eco-efficiency of families in the district of Ciudad Nueva.
\end{abstract}

Keywords: environmental awareness, eco-efficiency.

\title{
INTRODUCCIÓN
}

De acuerdo a lo que establecen Rincón y Chávez (2006) la conciencia ambiental es la convicción de una persona, una organización o un grupo social, de que los recursos naturales deben protegerse y usarse racionalmente en beneficio del presente y el futuro de la humanidad. Como tal, la conciencia ambiental está fundada en eco-valores que determinan la conducta o el comportamiento ecológico positivo. La importancia de la conciencia ambiental radica fundamentalmente en el hecho de que todas las actividades humanas generan efectos negativos en el ambiente; es decir, que ponen en grave riesgo el deterioro del equilibrio de los procesos naturales, lo cual afectará el desarrollo de todo tipo de vida. Para mitigar los efectos negativos sobre el ambiente, se han diseñado estrategias basadas en la ecoeficiencia,la cuales buscan mejorar los procesos productivos para aumentar la eficiencia en el uso de los recursos, de modo que se reduzca la generación de agentes contaminantes.La ecoeficiencia es beneficiosa porque implica producir mas con menos (Ruggeri, 2010, citado en Cabana, 2017). En el caso de los hogares, en conjunto generan un importante impacto ambiental, ya que en ellos se llevan a cabo acciones y procesos que implican el consumo de agua, el consumo de energía, el consumo de materias primas y productos elaborados. Cada hogar es una fuente de generación de residuos sólidos, aguas residuales, emisiones gaseosas y generación de contaminación acústica (Ministerio del Ambiente, 2009). El suministro de agua potable es fundamental para la salud, la industria y la agricultura. Las personas más vulnerables no cuentan con servicios de agua y a veces tienen que recorrer distancias muy largas para conseguirla, o pagar de 10 a 20 veces más por ella. Se estima que a nivel mundial más de 2,000 millones de personas carecen de acceso a los servicios básicos de agua y saneamiento(Organización de las Naciones Unidas, 2019). En el Perú, se estiman que entre 7 
y 8 millones de peruanos no tienen acceso al agua potable (Oxford Committee for Famine Relief, 2019). Por otro lado, la creciente y sostenida demanda mundial de energía ha generado un incremento de las emisiones de $\mathrm{CO} 2$, elevando el nivel de concentración a más de 415 partes por millón (ppm), la mayor concentración alcanzada en cualquier otro momento en los últimos 800,000 años, desde antes de la evolución del homo sapiens (Holthaus, 2019).La alta concentración de $\mathrm{CO} 2$ en la atmósfera promueve el calentamiento global y el agotamiento de los recursos naturales. La conciencia ambiental en las personas expresala convicción de que los recursos naturales deben protegerse y usarse racionalmente en beneficio del presente y del futuro (Rincón y Chávez, 2006), mientras que la ecoeficienciaestá está referida a producir más bienes y servicios con menos impacto ambiental por el uso más eficiente de los recursos (Ministerio del Ambiente, 2012). En Tacna diversos estudios como el de Manchego (2017), Churata (2017) y Cordova (2015), evidencian que los tacneños en su mayoría, alcanzan un nivel moderado de conciencia ambiental, mientras que la menor proporción alcanza un nivel alto de conciencia ambiental. Esta situación muestra que la población tacneña en general, cuenta con conocimientos del impacto que se está generando en su entorno debido a sus actividades diarias, sin embargo, no es suficiente este nivel de conocimiento para cambiar sus hábitos y actitudes para obrar bien sobre el medio ambiente (Vargas, Briones, Mancha, Múzquiz y Gaxiola, 2013). Considerando el distrito de Ciudad Nueva, 7,347 viviendas que representan al $88,79 \%$, tienen acceso al agua potable y 7,737 viviendas que representan al 93,5 \%, tienen acceso al servicio de electricidad (Municipalidad Distrital de Ciudad Nueva, 2016). La generación de residuos sólidos, en promedio en el distrito de Ciudad Nueva se genera 20,30 t/día y el distrito de Tacna produce 138,56 t/día (Córdova, 2015). A pesar de que el municipio ha implementado campañas de sensibilización para elevar la conciencia ambiental en la población del distrito de Ciudad Nueva, en temas referidos a la correcta gestión de los residuos domésticos (Diario Correo, 2016), no se observan evidencias de una correcta aplicación de criterios de minimización, segregación, almacenamiento, recolección y aprovechamiento de los residuos sólidos, por parte de los vecinos del distrito (Municipalidad Distrital de Ciudad Nueva, 2016). Aburto y Mardones (2017) aplicaron una metodología de enseñanza de la ecología. Demostró un aumento en la conciencia ambiental dentro del grupo experimental. Díaz y Fuentes (2018) analizaron el desarrollo de la conciencia ambiental en niños donde la dimensión afectiva es importante para promover el agrado, respeto y cuidado hacia la naturaleza. Herrera (2016) encontró que el nivel de conciencia ambiental es regular para el $60 \%$ de los estudiantes. Ore (2014) comprueba que los estudiantes mejoran su participación activa en el cuidado ambiental. Huamanyauri, Machaca y Peña (2014) la conciencia ambiental en los en los estudiantes de colegio. Vargas, Briones, Mancha, Múzquiz y Gaxiola (2013) afirma que si bien las personas generan actividades diarias consientes, sin embargo, no lo usa para obrar bien ante la Tierra. Gomera, Villamandos de la Torre y Vaquero (2012) midieron y categorizaron la conciencia ambiental de jóvenes universitarios y éstos no encuentran acciones positivas. Miranda (2017) encontró que no existe diferencia significativa entre los puntajes promedio de la conciencia ambiental en los estudiantes universitarios. Albarracin (2017 no observó correlación positiva en estudiantes universitarios. Vázquez (2008) refiere que cuando existe una mejora en el desempeño ambiental y económico de las empresas, mejora su nivel de ecoeficiencia, favorecido el aumento del nivel de gestión ambiental y de las utilidades. Calle (2017) encontró que existe una relación positiva entre el consumo de energía eléctrica y la Ecoeficiencia. Cabana (2017) también encontró que la conciencia ambiental y los valores inciden significativamente en la Ecoeficiencia de la Gerencia de Servicios. 
Conciencia ambiental significa conocer nuestro entorno para cuidarlo y que nuestros hijos también puedan disfrutarlo (Blanco, 2000, citado en Ore, 2014). Es la Convicción de una persona, organización, grupo o una sociedad entera, de que los recursos naturales deben protegerse y usarse racionalmente en beneficio del presente y el futuro de la humanidad (Rincón y Chávez, 2006). Ulloa (2017) establece que la conciencia ambiental es un entendimiento que se tiene del impacto de los seres humanos en el entorno. Cabana (2017) afirma que la conciencia ambiental es el conjunto de creencias, actitudes, normas, conocimientos y valores relacionados con el medio ambiente. La importancia de la conciencia ambiental radica fundamentalmente en el hecho de que todas las actividades humanas generan efectos negativos en el medio ambiente (Frers, 2011, citado en Cabana 2017). Tomando conciencia ambiental podemos evitar el aumento de contaminación en los espacios que residimos (Novo,1995).

\section{OBJETIVOS}

Determinar la influencia de la conciencia ambiental en el uso eficiente del agua, energía y en la gestión eficiente de residuos en las familias del distrito Ciudad Nueva.

\section{METODOLOGÍA}

La investigación siguió el enfoque cuantitativo. El ámbito del estudio correspondió al distrito de Ciudad Nueva, circunscripción perteneciente a la provincia de Tacna, Región Tacna y el horizonte social que abarcó fue el primer semestre del año 2019. La muestra fue probabilística, y se aplicó el muestreo aleatorio simple con un tamaño de 366 personas. La validación del instrumento de investigación se hizo mediante la técnica de juicio de expertos, y la confiabilidad del instrumento se determinó por aplicación de una prueba piloto, con un coeficiente alpha de cronbach de 0,815

\section{RESULTADOS}

El jefe de familia tiene una edad promedio de 40,6 años y se encontró que el ingreso promedio mensual de las familias es de S/. 1678,6 . El 29,8 \% de las familias tienen un tamaño familiar de 4 miembros. El nivel de actitud afectiva que se observó en el 49,7\% de los casos es de nivel regular. El nivel de actitud cognitiva en el $82,2 \%$ de los casos también es de nivel regular, así como el nivel de actitud conativa donde el $56,0 \%$ de los casos también es de nivel regular. El nivel de actitud activa que se observó en las familias del distrito de Ciudad Nueva, es en el 3,8 $\%$ de los casos, de nivel bajo; en el $64,8 \%$ de los casos, de nivel regular; en el 30,6 \% de los casos, de nivel alto y en el $0,8 \%$ de los casos, de nivel muy alto. El nivel de conciencia ambiental es en el 0,3 \% de los casos, de nivel bajo; en el 79,8 \% de los casos, de nivel regular y en el $19,9 \%$ de los casos, de nivel alto.

El nivel de uso eficiente del agua es en el 0,5 \% de los casos, de nivel muy bajo; en el 40,4 \% de los casos, de nivel bajo; en el $42,6 \%$ de los casos, de nivel regular (Figura 1). El nivel de uso eficiente de energía el 65,2 \% de las familias tienen a lo más un nivel regular de uso eficiente de energía y que el $34,8 \%$ de las familias, tiene al menos un nivel alto de uso eficiente de energía. (Figura 2). Respecto al nivel de gestión de residuos, el $86.3 \%$ de las familias tienen a 
lo más un nivel regular de gestión eficiente de residuos y que el $13,7 \%$ de las familias, tiene al menos un nivel alto de gestión eficiente de residuos (Figura 3).

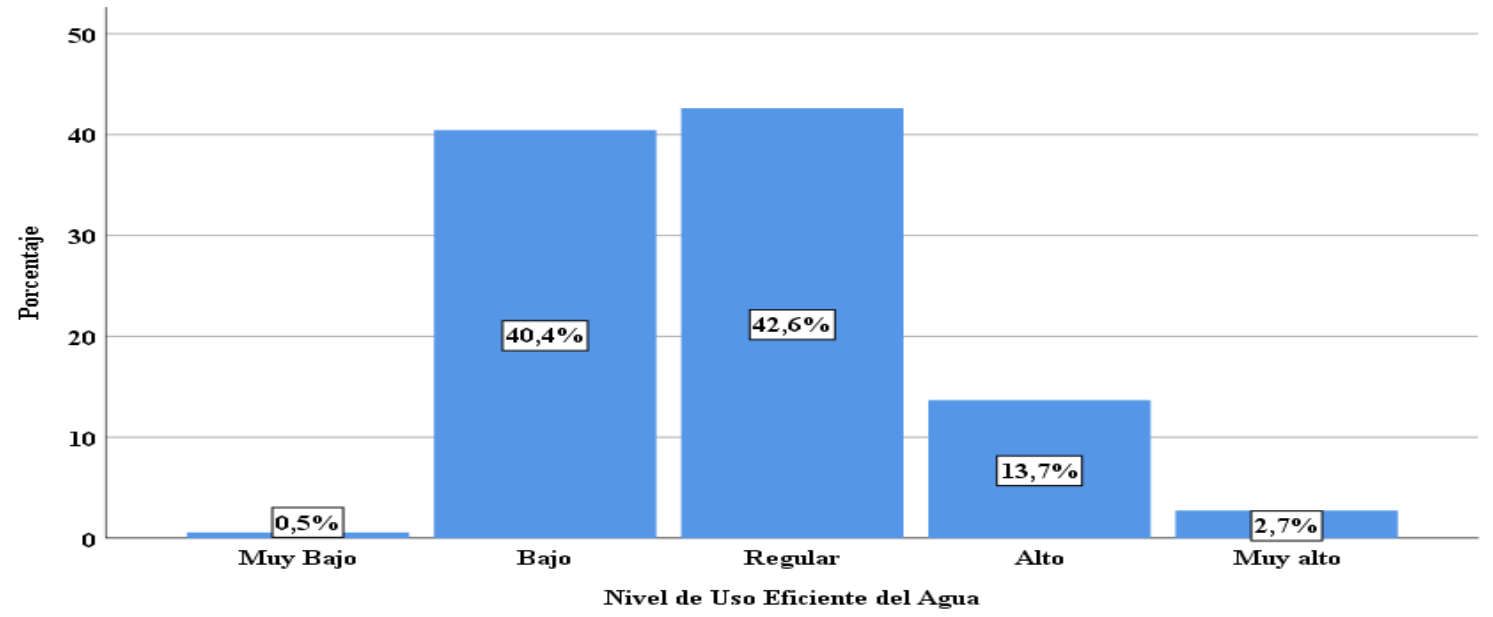

Figura 1. Nivel de uso eficiente de agua en las familias



Figura 2. Nivel de uso eficiente de energía en las familias

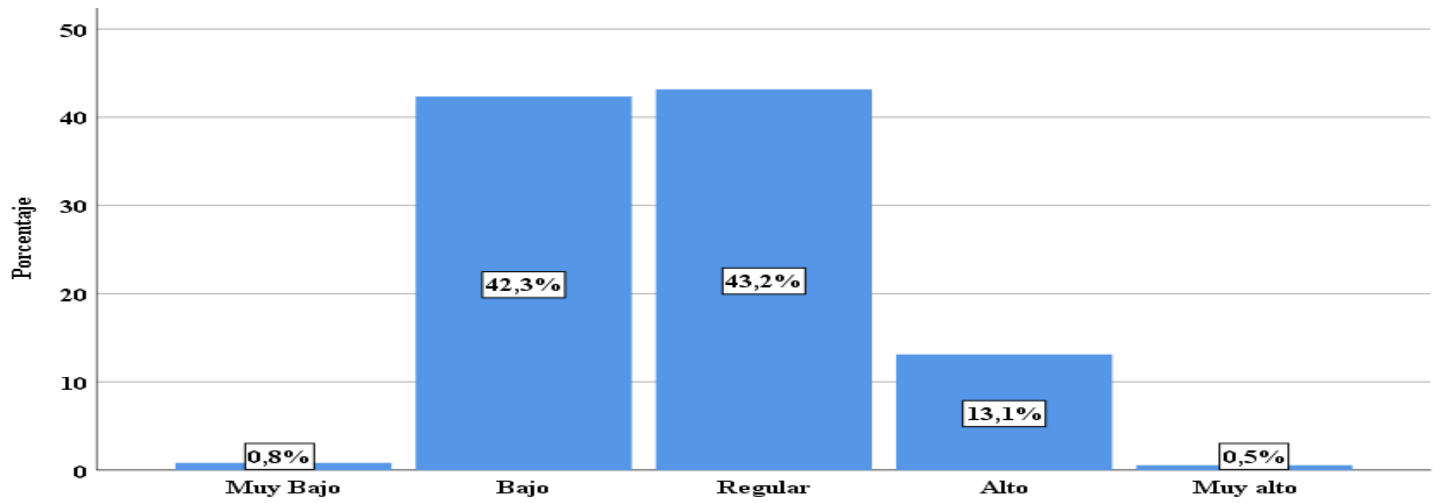

Nivel de Gestion Eficiente de Residuos

Figura 3. Nivel de gestión eficiente de residuos en las familias 
Respecto a los residuos sólidos reutilizables y no reutilizables, el 49,7 no separa los materiales que componen la basura con el fin de reciclarlos racionalmente. El 75,7 \% de las familias del distrito de Ciudad Nueva no utiliza los residuos de frutas, tubérculos y verduras para producir compost o abono casero. El resto de familias, es decir el $24,3 \%$, si utiliza los residuos de frutas, tubérculos y verduras para producir compost o abono casero. El 50,3\% no clasifica los residuos en metales papel o cartón, vidrio, plástico, residuos orgánicos y residuos peligrosos. El resto de familias, es decir el 49,7 \%, si clasifica los residuos en metales, papel o cartón, vidrio, plástico, residuos orgánicos y residuos peligrosos (tabla 01)

Tabla 01

Residuos Sólidos

\begin{tabular}{lcccc}
\hline & \multicolumn{2}{c}{ si } & \multicolumn{2}{c}{ no } \\
\cline { 2 - 5 } & $\mathbf{n}$ & $\mathbf{\%}$ & $\mathbf{n}$ & $\mathbf{\%}$ \\
\hline Separación de Materiales que Componen la basura & 184 & 50.3 & 182 & 49.7 \\
Utilización de Residuos para producir Compost & 89 & 24.3 & 277 & 75.7 \\
Clasificación de los Residuos & 182 & 49.7 & 184 & 50.3 \\
\hline
\end{tabular}

El nivel de ecoeficiencia que se observó en las familias del distrito de Ciudad Nueva, es en el $37,4 \%$ de los casos, de nivel bajo; en el $51,4 \%$ de los casos, de nivel regular; en el $10,7 \%$ de los casos, de nivel alto y en el $0,5 \%$ de los casos, de nivel muy alto. Estos resultados permiten afirmar que el $88,8 \%$ de las familias tienen a lo más un nivel regular de ecoeficiencia y que el $11,2 \%$ de las familias, tiene al menos un nivel alto de ecoeficiencia (Fig. 4)

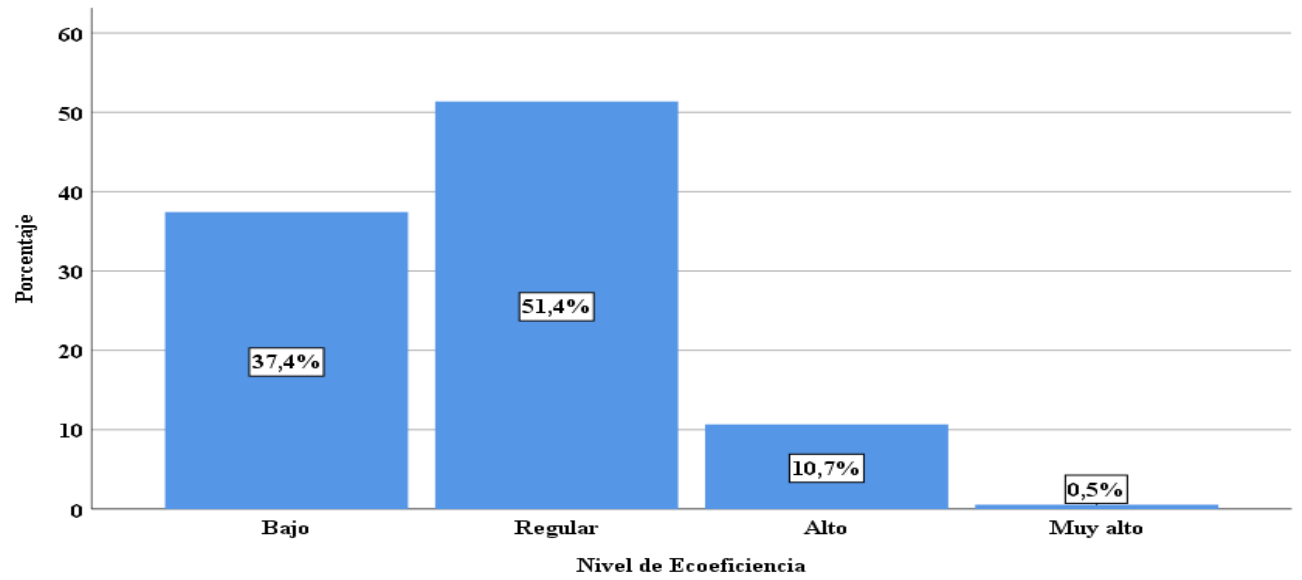

Figura 2. Nivel de ecoeficiencia de las familias

Se encontró débil correlación positiva entre las variables conciencia ambiental y uso eficiente del agua. La actitud afectiva influye negativamente en el uso eficiente del agua, sin embargo, la actitud cognitiva, conativa y activa influyen positivamente. Se encontró moderada correlación positiva entre las variables conciencia ambiental y uso eficiente de energía. La actitud afectiva no influye en el uso eficiente de energía, en cambio, la actitud cognitiva, conativa y activa influyen positivamente en el uso eficiente de energía en las familias del distrito de Ciudad Nueva con una seguridad estadística del $99 \%$. La actitud afectiva no influye 
en la gestión eficiente de residuos. La actitud afectiva influye negativamente en la ecoeficiencia, en cambio, la actitud cognitiva, conativa y activa influyen positivamente en la ecoeficiencia en el grupo de estudio, con una seguridad estadística del 99 \%. (Correlación Rho de Spearman)

\section{DISCUSIÓN}

Se encontró una correlación débil, positiva y altamente significativa entre las variables conciencia ambiental y ecoeficiencia $\left(r=+0,386^{* *}\right)$, una correlación débil, positiva y altamente significativa entre las variables conciencia ambiental y uso eficiente del agua $\left(r=+0,253^{* *}\right)$, una correlación moderada, positiva y altamente significativa entre las variables conciencia ambiental y uso eficiente de energía $\left(r=+0,418^{* *}\right)$ y una correlación débil, positiva y altamente significativa entre las variables conciencia ambiental y gestión eficiente de residuos $\left(r=+0,355^{* *}\right)$. Se recomienda profundizar la investigación, identificando en las familias del distrito de Ciudad Nueva, los grupos por edad, sexo, tamaño familiar e ingreso, que tienen menores niveles de conciencia ambiental y ecoeficiencia. Este estudio permitirá la implementación focalizada de programas de educación ambiental en el distrito de Ciudad Nueva. Ampliar la investigación analizando la influencia de la conciencia ambiental en la ecoeficiencia de las familias del distrito Tacna, de modo que se compare a través de variables intervinientes como el nivel de ingreso y nivel educacional, las similitudes y diferencias observadas con las familias del distrito de Ciudad Nueva. Extender la investigación tomando como unidad de estudio, una institución gubernamental, de modo que se pueda cuantificar el impacto económico del uso eficiente del agua, la energía y la gestión eficiente de los residuos de oficina, de acuerdo al nivel de conciencia ambiental que presentan los trabajadores de dicha institución. En los hogares la ecoeficiencia representa usar eficientemente el agua, la energía además de la gestión eficiente de los residuos. Desde el punto de vista económico, estas acciones representan una disminución en los gastos del servicio de agua y energía eléctrica y la obtención de ingresos extras por el reciclaje de residuos sólidos. En ese sentido, se recomienda ampliar la investigación cuantificando los beneficios económicos que representa aplicar la ecoeficiencia en las familias del distrito de Ciudad Nueva. La educación es un factor importante para elevar la conciencia ambiental. Es por ello que se recomienda profundizar la investigación a un nivel causal experimental, donde se diseñe y proponga un método educativo para elevar la conciencia en las familias del distrito de Ciudad Nueva.

\section{REFERENCIAS BIBLIOGRÁFICAS}

Aburto, P., \& Mardones, D. (2017). Aplicación de la metodología Enseñanza de la Ecología en el Patio de la Escuela, para aumentar la conciencia ambiental de los estudiantes pertenecientes a un establecimiento educacional de la comuna de Los Ángeles. Los Ángeles, Chile: Tesis (Titulo). Universidad de Concepción. p. 103.

Albarracin, N. (2017). El nivel de conocimiento sobre temas ambientales y la conciencia ambiental de los estudiantes del nivel avanzado (ciclo VII, VIII, IX Y X) de la carrera profesional de Arquitectura de la Universidad Privada de Tacna, 2015. Tacna: Tesis (Maestría). Universidad Privada de Tacna. p. 173.

Aznar, P. (2013). La responsabilidad por un mundo. España: Desclée Brounwer.(p.130). 
Cabana, A. (2017). Conciencia ambiental, valores y ecoeficiencia en la Gerencia de Servicios a la Ciudad y Medio Ambiente. Lima Cercado. 2016. Lima: Tesis (Doctorado). Universidad César Vallejo. p.141.

Calle, C. (2017). Relación entre el consumo de energía eléctrica y la ecoeficiencia en los hogares de la urbanización de Villa Sol, 2da etapa - Los Olivos, 2017. Lima: Tesis (Titulo). Universidad Cesar Vallejo. p.126.

Córdova, A. (2008). Influencia de la armonía del ambiente con flores en el nivel de logro del aprendizaje en el área de ciencia, tecnología y ambiente de los estudiantes del $1^{\circ}$ del nivel secundaria de la IE N 5124 Ventanilla - Callao. Lima-Perú.

Diario Correo. (16 de Agosto de 2016). Página web oficial del Diario Correo. Recuperado el 1 de Setiembre de 2018, de https://diariocorreo.pe/edicion/tacna/instalan-232-contenedores-debasura-en-diferentes-sectores-del-distrito-de-ciudad-nueva-690588/

Díaz, J., \& Fuentes, F. (2018). Desarrollo de la conciencia ambiental en niños de sexto grado de educación primaria. Significados y percepciones. Revista de Investigación Educativa, (26). 136163.

Fresco, P. (2018). El futuro de la energía en 100 preguntas. Madrid: Ediciones Nowtilus S.L. p.352 .

Gomera, A., Villamandos de la Torre, F., \& Vaquero, M. (2012). Medición y categorización de la conciencia ambiental del alumnado universitario: contribución de la universidad a su fortalecimiento. Revista Profesorado, 16 (2). 213-228. Recuperado el 3 de Setiembre de 2018, de https://www.ugr.es/ recfpro/rev162ART11.pdf

Herrera, J. (2016). Conciencia ambiental en los estudiantes del tercer grado de educación secundaria del colegio nacional Politécnico del Callao, 2016. Lima: Tesis (Titulo). Universidad Cesar Vallejo. p. 79.

Holthaus, E. (. (13 de Mayo de 2019). Página web oficial de CNN en español. Recuperado el 2 de Setiembre de 2019, de https://cnnespanol.cnn.com/2019/05/13/hay-mas-co2-en-la-atmosferahoy-que-en-cualquier-otro-momento-desde-la-evolucion-humana/\#0

Huamanyauri, R., Machaca, L., \& Peña, R. (2014). Manejo de residuos sólidos y su relación con la conciencia ambiental en los estudiantes del 2 do grado de secundaria de la Institución Educativa $N^{\circ} 119$ Canto Bello - San Juan de Lurigancho, 2014. Lima: Tesis (Titulo). Universidad Nacional de Educación Enrique Guzmán y Valle. p.89.

Instituto Nacional de Estadística e Informática del Perú. (2018). Página web oficial del INEI. Recuperado el 9 de Setiembre de 2018, de https://www.inei.gob.pe/estadisticas/indice-tematico/poblaciony-vivienda/

Ministerio del Ambiente. (2009). Página web de la PCM. Recuperado el 7 de Setiembre de 2018, de http://hera.pcm.gob.pe/ecoeficiencia/wp-content/uploads/2014/09/Guia_ecoeficiencia_SPMINAM-2009.pdf

Ministerio del Ambiente. (2010). Página web oficial del Gobierno Regional de Ica. Recuperado el 2 de Setiembre de 2018, de http://www.regionica.gob.pe/pdf/transparencia\%202010/otros/ecoeficiencia/Guia\%20de\%20 Ecoeficiencia\%20Educacional.pdf

Ministerio del Ambiente. (2012). Ciudadanía ambiental Guía de educación en ecoeficiencia. Lima: Editorial Giacomotti Comunicación Gráfica S.A.C. p.117.

Ministerio del Ambiente. (2012). Página web oficial de la Universidad San Matín de Porres. Recuperado el 15 de Enero de 2019, de http://www.usmp.edu.pe/recursoshumanos/pdf/Glosario-deTerminos.pdf

Ministerio del Ambiente. (2016). Página web oficial del MINAM. Recuperado el 7 de Setiembre de 2018, de http://www.minam.gob.pe/educacion/wpcontent/uploads/sites/20/2017/02/Publicaciones-2.-Texto-de-consulta-M\%C3\%B3dulo-2.pdf

Ministerio del Ambiente. (2019). Página web ofiacil del MINAM. Recuperado el 01 de Setiembre de 2019, de http://www.minam.gob.pe/gestion-de-residuos-solidos/norma-tecnica-peruana-decolores-ntp-900-058-2019/ 
Miranda, D. (2017). Medición de la conciencia ambiental en estudiantes de ciencias agrarias de la Universidad Nacional de Tumbes. Revista Humanidades e Inovação, 4 (2). 108-123.

Municipalidad Distrital de Ciudad Nueva. (Noviembre de 2016). Página web oficial de la MDCN. Recuperado el 1 de Setiembre de 2018, de http://www.municiudadnueva.gob.pe/webmdcn/documentos/PDLC\%202017-2021.pdf

Novo, M. (1995). La educación ambiental. Bases éticas, conceptuales y metodológicas. Madrid: Universitas.

Ore, W. (2014). Proyecto educativo institucional con enfoque ambiental para desarrollar conciencia ambiental en los estudiantes de la I. E. San Daniel Comboni. Huancayo: Tesis (Maestría). Universidad Nacional Del Centro Del Perú. p. 121.

Organización de la Naciones Unidas. (1987). Página web oficial de la ONU. Recuperado el 12 de Junio de 2019, de https://www.un.org/spanish/conferences/wssd/desarrollo.htm

Organización de las Naciones Unidas. (18 de Marzo de 2019). Página web oficial de la ONU. Recuperado el 2 de Setiembre de 2019, de https://news.un.org/es/story/2019/03/1452891

Oxford Committee for Famine Relief. (2019). Página web oficial de OXFAM. Recuperado el 2 de Setiembre de 2019, de https://peru.oxfam.org/qué-hacemos-ayuda-humanitaria/entre-7-y-8millones-de-peruanos-no-tienen-acceso-agua-potable

Paz, M. (2000). La educación ambiental en Chile. Chile. (p.323)

Quezada, N. (2017). Estadistica con SPSS 24. Lima: Empresa Editora MACRO. P. 446.

Ramos, R., Sepúlveda, R., \& Villalobos, F. (2003). El agua en el medio ambiente: muestreo y análisis. México: Plaza y Valdes. p.210.

Superintendencia Nacional de Servicios y Saneamiento. (30 de Noviembre de 2018). Página web oficial de la SUNASS. Recuperado el 1 de Setiembre de 2019, de https://www.sunass.gob.pe/websunass/index.php/sunass/regulaciontarifaria/doc_details/3356-eps-tacna-s-a-estudio-tarifario-2019-2024

Ulloa, J. (2017). Conservación del ambiente por los estudiantes de la facultad de educación de la Universidad Nacional de Huancavelica. Huancavelica: Tesis (Maestría). Universidad Nacional de Huancavelica. p.132.

Vargas, C., Briones, C., Mancha, M. d., Múzquiz, P., \& Gaxiola, A. (2013). Conciencia ambiental de los habitantes de la colonia Emilio Portes Gil en la H. Matamoros, Tamaulipas. Revista Luna Azul de la Universidad de Caldas, (37). 155-161. Recuperado el 4 de Setiembre de 2018, de http://www.scielo.org.co/scielo.php?pid=S1909-

24742013000200011\&script=sci_abstract\&tIng=es

Vázquez, A. (2008). Análisis de la Ecoeficiencia en la Producción del Mezcal. Sta. Cruz Xoxocotlán, Oaxaca, México: Tesis (Maestria). Instituto Politécnico Nacional. p. 109. 